\title{
UML Model Applied as a Useful Tool for Wireless Body Area Networks
}

\author{
Giovanni Saggio $^{\# * 1}$, Pietro Cavallo ${ }^{\# 2}$, Luigi Bianchi ${ }^{\S *}{ }^{* 3}$ Lucia R. Quitadamo $^{\S \& 4}$, Franco Giannini $^{\# * 5}$ \\ \#Department of Electronic Engineering, “Tor Vergata” University, via del Politecnico 1, 00133 Rome, Italy \\ ${ }^{\S}$ Department of Neuroscience, “Tor Vergata” University, via Montpellier 1, 00133 Rome, Italy \\ "Centro di Biomedicina Spaziale, “Tor Vergata” University, via del Politecnico 1, 00133 Rome, Italy \\ ${ }^{\&}$ Department of Neurophysiology, Fondazione S. Lucia, IRCCS, via Ardeatina 306, 00179 Rome, Italy \\ saggio@uniroma2.it
}

\begin{abstract}
Body Area Network (BAN) and Wireless BAN (WBAN) systems lack a unique description model for the identification of all the components and the features that characterize them. This results in a disadvantage since a formalization tool would favor standardization and would seriously help in the implementation of building systems. In this paper we successfully used the Unified Modeling Language (UML) to describe a WBAN which measures hand joint movements, and we demonstrated how UML can be successfully adopted to design a model for the description of such networks.
\end{abstract}

Keywords- UML, BAN, WBAN

\section{INTRODUCTION}

$\mathrm{F}$ OR hand medical rehabilitation purposes it would be useful to count on a system which continuously measures finger joint movements. To this aim we developed an adhoc instrumented glove based on bend sensors which convert joint angle value into electrical signal. Measured data are then pre-processed, binary converted, and wireless transmitted to a personal computer base station. Received data are then utilized to implement a virtual reality environment which graphically reproduces real human finger movements.

In this work we adopted the Unified Modeling Language as a useful tool capable of describing our system of hand joint movement data acquisition, condition and exploitation.

The purpose is to stress how UML can be used as a powerful modeling instrument for describing Body Area Network systems which can be similar or quite different [1], [2] from our application.

The first stage of our WBAN system consisted of an instrumented glove realized with a lycra based material as support of 19 bend sensors, one for each finger's degree of freedom, including flex-extension and adduction-abduction movements. Every sensor consists of a film resistor that changes its resistance when bended.

This work was partially supported by the DCMC Project of the Italian Space Agency. This paper only reflects the authors' views and funding agencies are not liable for any use that may be made of the information contained herein.

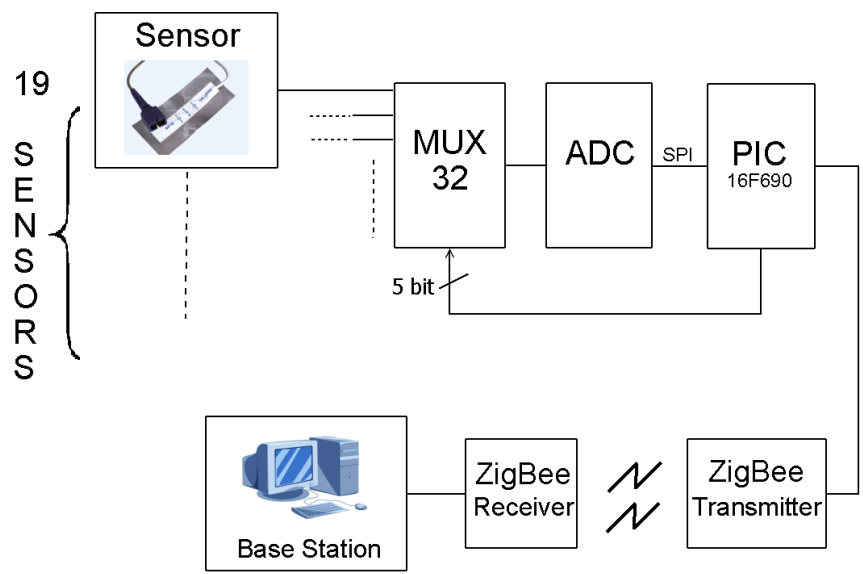

Figure 1. Block diagram for the hand joint movement data acquisition system

The second system's stage regarded the circuitry. Thanks to a voltage divider applied to every sensor we get the corresponding voltage which was properly scaled between 0 and 5 Volts. Voltage values were then passed to a multiplexer. One analog value at time was converted into a digital one and furnished to a PIC via SPI protocol. The PIC provided read values to a wireless network based on ZigBee protocol.

The wireless network, considered as the third stage, had a classic star-shaped topology, in which sensors represent the so called edge-nodes, while the set of MUX, ADC, PIC and wireless transmitter represent the central-node.

As a final stage, the information were transferred to a personal computer that interpreted, classified and utilized them. In particular a home-made software implemented a virtual reality situation replying in animation the real finger movements.

The entire system is schematically reported in fig. 1 .

In the next paragraphs we will use both static and dynamics diagrams to represent the structure of the entire system and the interaction within the system's components. We will use UML class diagrams and sequence diagrams because they are very intuitive and can capture complex aspects of a generic WBAN system. 


\section{METHODS}

UML is a widespread visual language provided by Object Management Group for the description, documentation and modeling of systems, software and processes. A well-designed UML model can be easily translated into real world applications.

UML was already successfully adopted for describing Brain Computer Interface (BCI) systems, which are assistive devices which rely on the translation of brain signals into commands towards external peripherals to allow the communication. UML was also applied to Human-Computer Interaction generic systems; therefore for its definition and description we refer to previous works [3], [4],[5].

In this paper we used class diagrams and sequence diagrams for the identification of the major components of WBAN systems and their temporal relationship, in particular referring to our implemented system.

\section{A. A General System Architecture}

The following figure shows a high-level generic model of a system for acquisition and manipulation of biological signals.

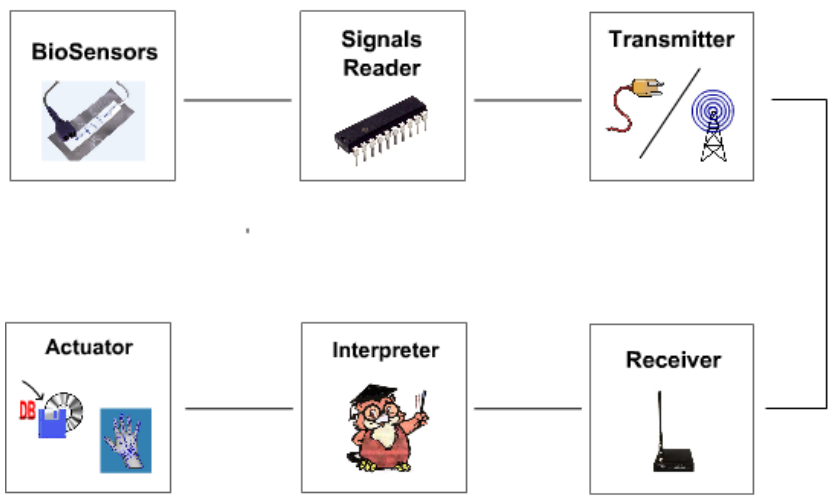

Figure 2. High-level model of a generic BAN system

Acquisition block reads bio-sensors' signals, eventually formats or ciphers them and sends signals to the transmitter. Data transmission could be wired or wireless and a receiver accepts signals for an interpreter which take care of syntactical correctness and, optionally, semantic interpretation (for example extracting key features, classifying them, etc.).

Data can be forwarded to external networks or can be utilized for controlling applications of virtual reality, for moving mechanical devices or, simply, for storing purposes.

\section{B. A General Class Diagram}

In order to formally describe WBAN systems, a general purpose UML Class Diagram has been here developed. The class diagram is schematically reported in fig. 3, and represents the structural part of the entire UML system (which must be integrated by the Sequence Diagram described in next paragraph). Here we want to demonstrate its validity with respect to the above mentioned WBAN system, pointing out correspondences between the real system and the involved class diagram blocks.

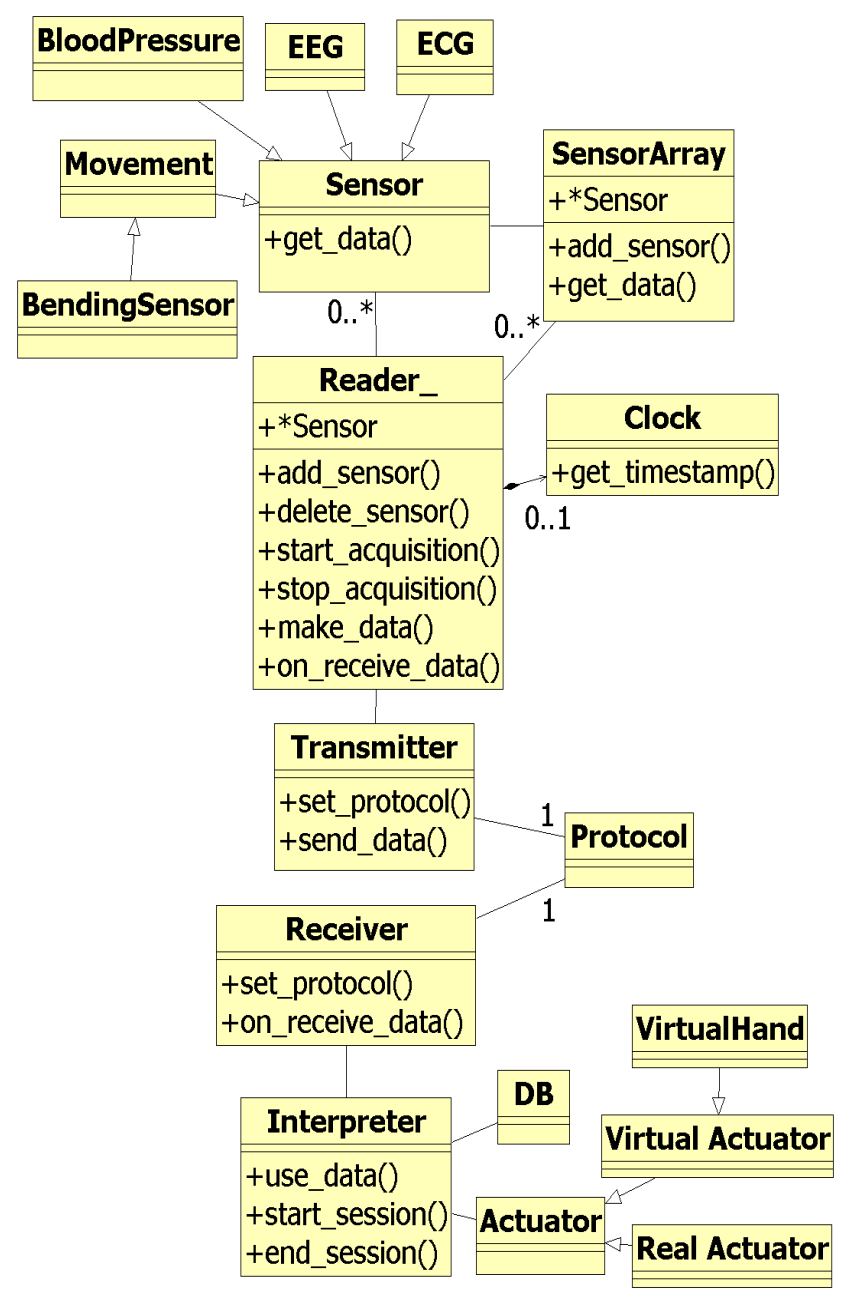

Figure 3. The here proposed general Class Diagram for WBAN systems

Class diagrams belong to the family of structural diagrams as they represent the constitution's scheme by means of classes which are the components of the system itself. A class is a category or a set of objects that share the same attributes, operations, relationships and semantics.

Each class diagram logic block is represented with a rectangular box divided into compartments. The first compartment contains the name of the class, the second contains the attributes, which are the details of the class and represent its internal states and the third contains the operations, or methods, which are the particular behaviours of the class.

Interactions within classes are represented as relationships through connections.

Paying particular attention on the Sensor class, it defines an abstraction of any kind of sensor. The surrounding child classes (which can be of several kinds, as examples: EEG, ECG, BloodSugar, etc.) pointing to Sensor (their "father") by means of thick arrows, inherit full characteristics and define Sensor's class specialization.

SensorArray class was also created to model those sensors which are read in block. 
Other classes model the generic system's blocks seen before, and will be explained in details in the next paragraph.

\section{Mapping the general purpose Class Diagram to our System}

As previously mentioned, our system was made of several stages which can be almost directly mapped into our model's blocks.

According to the schematic representation of our system as in fig. 2, let's report the mapping, assuming as a starting point the bio-sensors block. Such block finds its UML correspondence with the BendingSensor class, which is a specialization of the Movement class, which is, again, a specialization of the father Sensor class. In particular we adopted ink carbon based commercial bend sensors, provided by Image SI, capable to transduce mechanical movements into electrical signals, within the limit of $0-80 \% \Delta R / R$ values. In our system, signals were read by an electronic circuitry previously described which includes a multiplexer, an $\mathrm{A} / \mathrm{D}$ converter and a PIC microcontroller. The sum of these devices belongs to the Reader class which in turn is capable of several actions further detailed in the Sequence Diagram. Related to the developed system the resistive values were converted into electric potential within $0-5 \mathrm{~V}$ and then digitally transformed into $2^{12}$ values thanks to a 12 bit $\mathrm{A} / \mathrm{D}$ converter.

Connected to the PIC there is a wireless transmitter, ZigBee protocol based. With respect to the Class Diagram it was mapped through the Transmitter class. As a next stage of our system there was a wireless receiver, in UML associated with the Receiver class. The wireless transmitter can assure a stream of $78120 \mathrm{bit} / \mathrm{sec}$ with a battery autonomy of $72 \mathrm{~h}$.

In our UML model Transmitter class and Receiver class communicate through the Protocol class which sets all the ZigBee parameters (data rate, acknowledgment, etc.).

All data were received by a personal computer on which a software block was involved in interpreting received data, formatting information and associating it to actions. This block corresponds to the Interpreter class.

Interpreter provides data to another software block, a virtual reality actuator. This is an application that displays on screen a 3D model of the human hand moving fingers according to the received data (fig. 4). In the UML model this corresponds to VirtualHand class, which is a specialization of VirtualActuator class, the child of the general class Actuator.

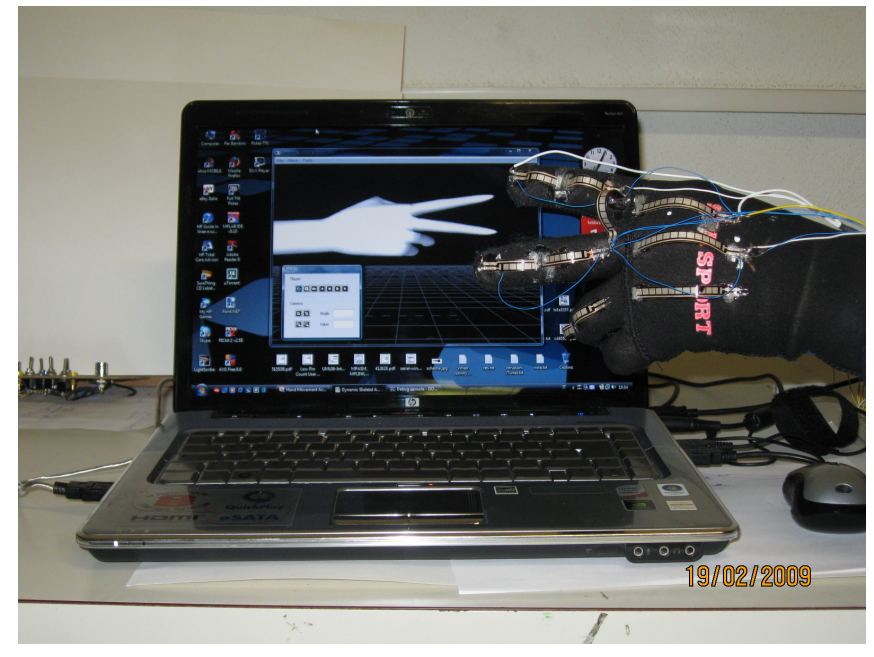

Figure 4. Virtual hand moving like the real hand. According to our model virtual hand software is mapped into the Actuator class.

\section{Modeling system behavior: the Sequence Diagram}

The UML Sequence Diagram is shown in fig. 5. It represents the timing of the events succeeding during system's life. The blocks on the top represents the actors of the system, called objects. Before the beginning of the session, all actors are instantiated by the so called Main Program. During the execution they communicate among each other using operations, represented as an arrow pointing from the subject of the action to the actor who suffers the action.

On the left a time line depicts a well-defined temporal sequence divided in three parts: Initialization, Running and End Session.

According to our system, in the Initialization phase we choose the number of sensors (19) and let the reader recognize them. In the UML Sequence Diagram this corresponds to the add_sensor() operation applied to the Reader. Now the protocol must be defined so the user must set all the ZigBee parameters in the UML Protocol object.

Following, the ZigBee protocol (set protocol()) must be applied both to the Receiver and to the Transmitter letting them communicate.

In the Running phase reader block gets data from each sensor and, if requested, formats the data eventually in association with a timestamp. In the Sequence Diagram these actions were performed through get_data(), get_timestamp() and make_data() operations.

After that, the Reader provides data to the Transmitter that carries information via wireless to the Receiver object. 


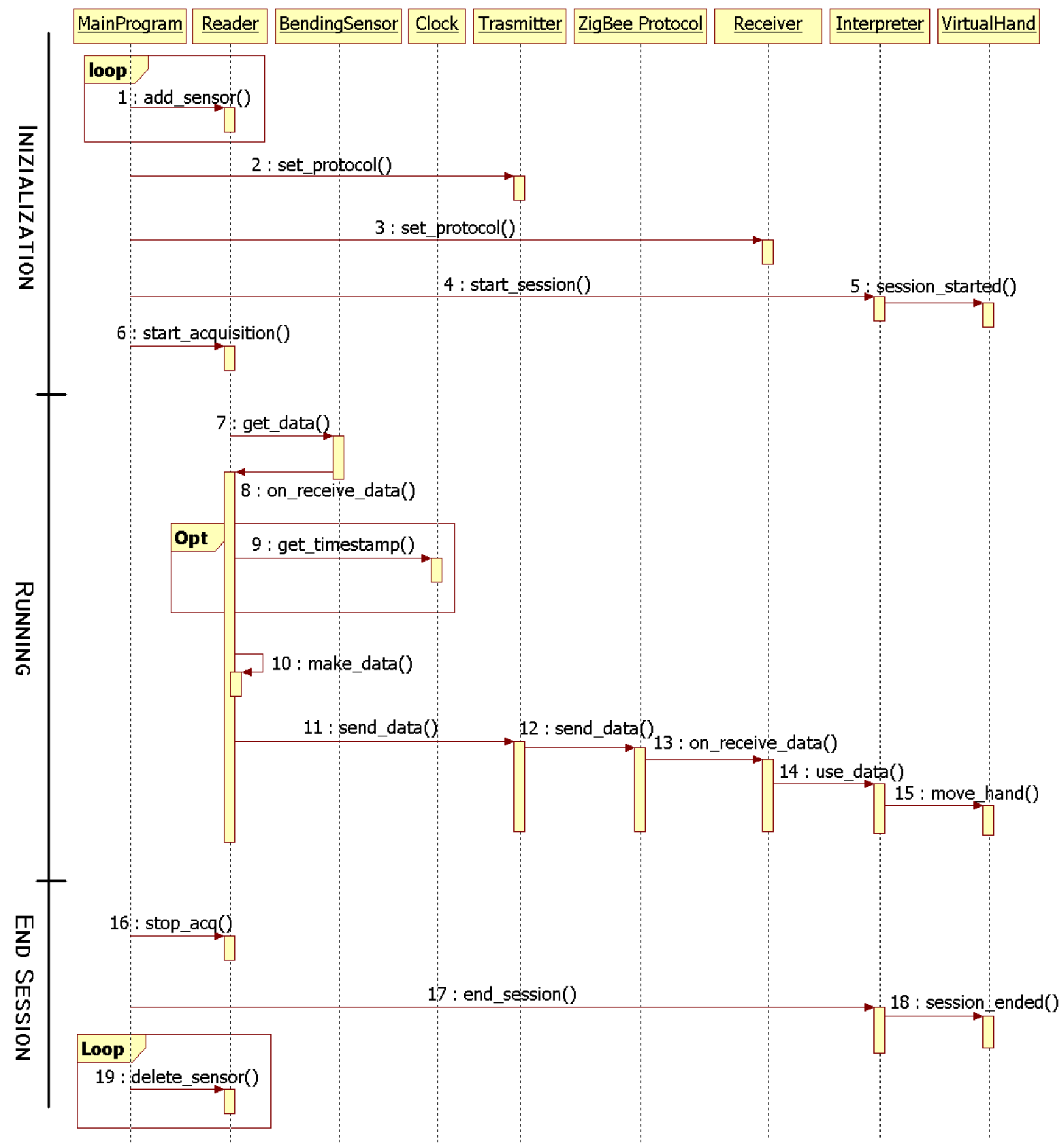

Figure 5. Sequence Diagram of the hand joint movement data acquisition system

Send_data() operates to lead data from the Receiver to the Transmitter object. Transmitter communicates to the Protocol again through send_data() operation. Now Protocol gives data to the Receiver actor via the on_receive_data() function.

The Interpreter gets data and manipulates it, classifying hand movements and furnishing them to the final application. In Sequence Diagram the Reader object calls the use_data() operation on the Interpreter actor. Finally Virtual Hand application uses data to visualize a $3 \mathrm{D}$ hand model moving according with real measured finger movements. This is realized with the move hand() operation invoked from the Interpreter object on the $\bar{V}$ irtualHand actor.

The last phase regards the end of the session. The system should stop the acquisitions, turning off the software blocks 
which were running on the PC. So in the sequence diagrams corresponded actors (Interpreter and VirtualHand) are stopped with stop_acq() and end_session() operations.

\section{CONCLUSION}

In this paper we reported how the UML tool can furnish a useful description model for Wireless Body Area Networks. WBAN technology is being widely researched and once accepted and adopted is expected to be a breakthrough innovation in healthcare.

After a brief description, UML was successfully applied to an our specific system, consisting in measuring, preprocessing, wireless transmitting, post-processing data from sensors utilized to measure human finger joint movements.

The paper demonstrated how the UML can completely describe a WBAN system.

We chose to describe our model by means of UML because in this way it is quite intuitive and can be easily modified and adapted according to the system requirements.

Our first attempt was to show that it is possible to create a standard model to represent WBAN systems that allows common approaches to similar problems. This results in a advantage for the implementation of new systems. Indeed, similar networks can reuse some resources already defined. A standard definition can finally allow an easy communication between different developers since they can use a common vocabulary and terminology for describing their systems.

\section{REFERENCES}

[1] E. Jovanov, A. Milenkovic, C. Otto, P. C de Groen "A wireless body area network of intelligent motion sensors for computer assisted physical rehabilitation" Journal of NeuroEngineering and Rehabilitation, 2005, vol. 2:6

[2] E. Jovanov, A. Milenković, C. Otto, P. De Groen, B. Johnson, S Warren, G. Taibi "A WBAN System for Ambulatory Monitoring of Physical Activity and Health Status: Applications and Challenges" Proceedings of the 27th Annual International Conference of the IEEE Engineering in Medicine and Biology Society, Shanghai, China, September 2005

[3] L.R. Quitadamo, M.G. Marciani, G. Cardarilli, L. Bianchi "Describing different Brain Computer Interface Systems through a unique model: a UML implementation" Neuroinformatics vol. 6:2, pp. 81-96

[4] L.R. Quitadamo, M. Abbafati, G. Saggio, M.G. Marciani, G.C.Cardarilli, L. Bianchi "A UML model for the description of different BCI systems" Engineering in Medicine and Biology Society, 30th Annual International Conference of the IEEE, pp. 1363-1366

[5] Z. Obrenovic, D. Starcevic "Modeling Multimodal Human-Computer Interaction" Computer, 2004, vol. 37, pp. 65- 72, 0018-9162. 